\title{
Dietas con diferentes fuentes lipídicas: su efecto en el perfil de ácidos grasos séricos de la rata
}

\author{
Diets with different lipid sources: \\ their effect on fatty acid serum \\ profile of the rat
}

\begin{abstract}
TThe importance of diet in maintaining health is widely accepted and recognized. Diet lipid profile is important to prevent chronic diseases and improve the quality of life of individuals. The objective of this study was to analyze the effect of different sources of dietary lipids with normal and high concentration, on the fatty acid profile of growing rats. Experimental diets contained 15 and $40 \% \mathrm{kcal}$ from fat, provided by butter (B), olive oil $(O)$, high oleic acid sunflower oil (SO) and sunflower oil (S). Control diet (C) was normocaloric, with $15 \% \mathrm{kcal}$ of fat provided by soy oil. All diets were complete in the others nutrients and were administered for 40 days. Daily intake was similar in all groups. $B, O$ and $S O$ groups had increased serum oleic acid and a decrease in serum essential fatty acids with respect to $C$. These results are seen independent of the fat proportion ( $F \%$ ) of the diet. S group showed an increase in linoleic acid only when F\% was 40. The administration of these diets provoked changes in serum fatty acid profile levels in response to the differences sources of dietary lipids used; only some changes observed in $S$ group were in response to the high fat percentage.

Key words: Nutrition; fatty acids; lipids; oils; butter; rats.
\end{abstract}

La importancia de la dieta en el mantenimiento del estado de salud es ampliamente aceptada y reconocida. A nivel mundial están aumentado rápidamente la carga de las enfermedades no transmisibles, la alimentación poco saludable y la falta de actividad física, que son las principales causas de enfermedades cardiovasculares, diabetes de tipo 2 y determinados tipos de cáncer. La Estrategia Mundial sobre Régimen Alimentario, Actividad física y Salud propone respecto a la dieta: lograr un equilibrio energético y un peso normal; limitar la ingesta energética procedente de las grasas, sustituir las grasas saturadas por grasas insaturadas y tratar de eliminar los ácidos grasos trans; aumentar el consumo de frutas y hortalizas, así como de legumbres, cereales integrales y frutos secos; limitar la ingesta de azúcares libres; limitar la ingesta de sal (sodio) (1).

Los niños y adolescentes con sobrepeso y obesidad tienen un mayor riesgo de enfermedades cardiovasculares. Existe una estrecha correlación entre la obesidad durante la niñez y la obesidad durante la vida, la cual aumenta con la edad. La prevención de la obesidad desde la niñez disminuiría en forma
Paula Daniela Perris Carolina Silva Inés Fernández María Cecilia Mambrin Nora H. Slobodianik María Susana Feliu

Cátedra de Nutrición. Universidad de Buenos Aires. Buenos Aires, Argentina.

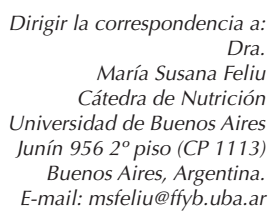

Este trabajo fue recibido el 23 de Enero de 2014 y aceptado para ser publicado el 29 de Junio de 2014.

significativa la morbimortalidad del adulto (2-4).

Los lípidos dietarios, los que deben ser consumidos en proporción adecuada, cumplen un rol muy importante en la alimentación. A partir de una publicación realizada en 1929 por George and Mildred Burr (5), los lípidos fueron dejados de ver como una simple fuente de energía. Las investigaciones mostraban que si se administraba una dieta carente de lípidos, las ratas se enfermaban y mayoritariamente terminaban muriendo. Luego, siguieron varias investigaciones las cuales permitieron conocer nuevas funciones. Hoy en día los lípidos son reconocidos como la fuente de energía más concentrada; fuente de ácidos grasos esenciales (AGE), vehículo de vitaminas liposolubles (vitaminas A, D, K y E), confieren palatabilidad a los alimentos, producen saciedad y cumplen un importante rol inmunomodulador.

Es fundamental un adecuado perfil de ácidos grasos (AG) para prevenir enfermedades crónicas y mejorar la calidad de vida de los individuos (6-11).

Los AG de las familias omega 3 y omega 6 presentan diferentes funciones de importancia en el organismo: participan en la formación de fosfolípidos de membrana, actúan como 
precursores en la síntesis de las prostaglandinas, tromboxanos, leucotrienos, prostaciclinas, todos ellos compuestos con funciones biológicas determinadas, pudiéndose mencionar entre ellas: la regulación de la tensión arterial, agregación plaquetaria, modulación de procesos inflamatorios, entre otros. Los omega 3 son protectores de la salud cardiovascular: disminuyen la concentración sanguínea de triglicéridos y colesterol, son débiles agregantes plaquetarios, previenen arritmias y mejoran la microcirculación. El consumo de AG de la familia omega 6 disminuye el nivel de colesterol total y las lipoproteínas de baja densidad (LDL). (8, 12, 13). Las familias omega 3, omega 6 y omega 9, comparten la misma ruta biosintética, utilizando las mismas enzimas (desaturasas y elongasas). De las tres series, la familia $\omega 3$ es la que presenta la mayor afinidad por las mismas, sin embargo, altos niveles de ácido linoleico $(\mathrm{AL})$ pueden inhibir la conversión de ácido alfa linolénico (AAL) en eicosapentaenoico (EPA) y docosahexaenoico (DHA). FAO-OMS recomienda que la dieta tenga una relación $\omega 6 / \omega 3$ entre 5 a 10 . Sin embargo en la mayor parte de los países industrializados de occidente, se consume una dieta muy desequilibrada en favor de los AG de la familia $\omega 6$ (relación $\omega 6 / \omega 3=20: 1$ ); esto mismo se observa en nuestro país, con un elevado consumo de aceite de girasol. Por otra parte, el tipo y la cantidad de AG de la dieta consumida habitualmente por el hombre, tiene un efecto directo tanto en la concentración de los lípidos plasmáticos como de las distintas lipoproteínas (14 - 17).

Teniendo en cuenta lo expuesto y debido a la importancia de la cantidad y el equilibrio de los lípidos en la dieta sobre el progreso de las enfermedades cardiovasculares y la obesidad, el objetivo de este trabajo fue analizar el efecto de dietas conteniendo, distintas fuentes lipídicas y diferente contenido calórico, durante 40 días, sobre el perfil de AG séricos, de la rata en período de crecimiento activo.

\section{MATERIALES Y MÉTODO}

Ratas bien nutridas durante la lactancia (6-8 crías por madre) se destetaron al llegar a un peso corporal entre 35-40 gramos (21-23 días de edad), momento a partir del cual fueron alimentadas durante 40 días con dietas conteniendo 15 ó 40 Kcal de lípidos en $100 \mathrm{Kcal}$ totales (F\%). Las fuentes lipídicas para ambas concentraciones fueron:

1. manteca/grasa láctea (dieta $M)$

2. aceite de oliva (dieta $\mathrm{O}$ )

3. aceite de girasol alto oleico (dieta AO)

4. aceite de girasol (dieta $G$ )

Como controles bien nutridos se utilizaron ratas que desde el destete recibieron dieta normocalórica (F\%=15 fuente lipídica: aceite de soja) y completa en todos los demás nutrientes según recomendaciones internacionales (dieta C). (18)

La composición de las dietas se presenta en la tabla 1.

En las experiencias se utilizaron 6-8 animales por lote, fueron realizadas por duplicado y los resultados corresponden al promedio de las mismas. Los animales fueron pesados al comienzo y al final de cada período experimental.

De las formas de alimentación utilizadas comúnmente en nutrición experimental se ha seleccionado para este trabajo la técnica de alimentación "ad libitum". En ésta se ofrece a los animales una cantidad de dieta superior a la que pueden consumir; y se determina la ingesta voluntaria después de un lapso determinado por pesada remanente. En todos los lotes experimentales se determinó el consumo de dieta, cada 2 - 3 días.

En todas las experiencias se utilizaron ratas de la cepa Wistar, de colonia cerrada del bioterio de las Cátedras de Bromatología y Nutrición de la Facultad de Farmacia y Bioquímica. Las ratas se alojaron individualmente en jaulas de acero galvanizado de piso de malla.

El perfil de AG de las dietas se determinó por cromatografía gaseosa (CG) y se calcularon las relaciones ácidos Grasos $\omega 6 / \omega 3$ y ácidos grasos poliinsaturados/ácidos grasos saturados (AGPI/AGS).

Al comienzo y al final de cada período experimental, los animales fueron pesados calculándose el aumento de peso diario, que fue posteriormente expresado como velocidad de ganancia ponderal (VGP)

Al final del período experimental, los animales (61 a 63 días de edad) se mantuvieron 3-4 horas en ayuno, y se aneste-

TABLA 1

Composición de las diferentes dietas experimentales.

\begin{tabular}{|c|c|c|c|c|c|c|c|c|c|}
\hline g/1000g de dieta & $\begin{array}{c}M \\
\mathrm{~F} \%=40\end{array}$ & $\begin{array}{c}M \\
\mathrm{~F} \%=15\end{array}$ & $\begin{array}{c}\mathrm{O} \\
\mathrm{F} \%=40\end{array}$ & $\begin{array}{c}\mathrm{O} \\
\mathrm{F} \%=15\end{array}$ & $\begin{array}{c}\mathrm{AO} \\
\mathrm{F} \%=40\end{array}$ & $\begin{array}{c}\mathrm{AO} \\
\mathrm{F} \%=15\end{array}$ & $\begin{array}{c}\mathrm{G} \\
\mathrm{F} \%=40\end{array}$ & $\begin{array}{c}G \\
F \%=15\end{array}$ & $\begin{array}{c}C \\
F \%=15\end{array}$ \\
\hline Caseína & 200 & 200 & 200 & 200 & 200 & 200 & 200 & 200 & 200 \\
\hline Sales & 35 & 35 & 35 & 35 & 35 & 35 & 35 & 35 & 35 \\
\hline Vitaminas & 10 & 10 & 10 & 10 & 10 & 10 & 10 & 10 & 10 \\
\hline Manteca & 225 & 70 & - & - & - & - & - & - & - \\
\hline Aceite de oliva & - & - & 225 & 70 & - & - & - & - & - \\
\hline Aceite girasol alto oleico & - & - & - & - & 225 & 70 & - & - & - \\
\hline Aceite girasol & - & - & - & - & - & - & 225 & 70 & - \\
\hline Aceite de soja & - & - & - & - & - & - & - & - & 70 \\
\hline Vit. A & 1 & 1 & 1 & 1 & 1 & 1 & 1 & 1 & 1 \\
\hline Cloruro de colina & 7,1 & 7,1 & 7,1 & 7,1 & 7,1 & 7,1 & 7,1 & 7,1 & 7,1 \\
\hline Dextrina & 521,9 & 676,9 & 521,9 & 676,9 & 521,9 & 676,9 & 521,9 & 676,9 & 676,9 \\
\hline
\end{tabular}


siaron con ketamina/clorhidrato de xilazina. Se extrajo sangre entera por punción cardíaca, se centrifugó y en el suero se determinó el perfil de ácidos grasos por CG previa extracción de los lípidos para obtener los metilésteres derivados de los ácidos grasos. Los ácidos grasos se identificaron de acuerdo a su tiempo de retención. Los resultados obtenidos se expresaron en porcentajes de ácidos grasos totales, tomándose como límite de cuantificación un valor de 0.05\%. (\% área media $\pm \mathrm{DE}$ ) (19). El análisis de la información obtenida se realizó utilizando ANOVA y DUNNETT como post test (20), considerando significativas las diferencias con el grupo control cuando $\mathrm{p}<0.01$.

\section{RESULTADOS}

$\mathrm{Al}$ analizar los cromatogramas de las dietas, se observaron diferencias en su composición. La dieta $M$ ofrece un mayor aporte de ácido Palmítico (26,4\% área total) en comparación con las otras dietas (con contenido menor al 11\%). Las dietas O y AO contienen un alto porcentaje de oleico (69\% y $85.3 \%$, respectivamente); la dieta $\mathrm{G}$ contiene una alta concentración de ácido linoleico (57,5\%), al igual que la dieta C $(53,31 \%)$. La dieta $C$ es la que tiene mayor aporte de ácido linolénico (5,92\%).

A partir de los datos obtenidos se calculó la relación $\omega 6 /$ w3 y la relación AG Poliinsaturados/AG Saturados (AGPI/AGS). FAO-OMS recomienda que por cada unidad de AG $\omega 3$ aportados por la dieta, deberían consumirse de 5 a 10 unidades de $\omega 6$. La relación $\omega 6 / \omega 3$ de las dietas $O(49,6) ; A O(86)$ y G (250) se encuentran por encima del valor del control, mientras que las dietas $M(5,6)$ y $C(9,0)$ se encuentran dentro del rango recomendado. La relación AGPI/AGS para las dietas $M(0,06)$, $\mathrm{O}(1,36)$ y $\mathrm{AO}(0,72)$ están disminuídas (valor recomendado $>1,5)$, ya que la dieta $M$ aporta gran cantidad de AGS y las dietas $\mathrm{O}$ y $\mathrm{AO}$ aportan baja proporción de AGPI.

El consumo de las dietas durante 40 días por los diferentes grupos y VGP de las ratas se presenta en la tabla 2.

No se hallaron diferencias significativas comparando cada grupo con el control correspondiente cuando se expresa el consumo en g/día. Tampoco se hallaron diferencias significativas en la VGP.

En la tabla 3 se presenta el perfil de ácidos grasos en suero de los grupos experimentales cuando las dietas fueron administradas por 40 días.

El grupo $M$ presenta aumento de los ácidos palmítico y oleico, con disminución de los AGE con respecto al grupo C. Los grupos experimentales $\mathrm{O}$ y $\mathrm{AO}$ presentan aumento de ácido oleico con disminución de ácidos linoleico, linolénico,y DHA. El grupo G muestra una disminución de ácido linolénico y DHA. Estos resultados se observan independiente del F\% de la dieta.

El grupo G muestra aumento de ácido linoleico sólo cuando la dieta contiene un F\%40.

\section{DISCUSIÓN Y CONCLUSIONES}

Las dietas tuvieron una buena aceptación por parte de las ratas y el consumo expresado como g/día de los diferentes grupos no difiere del grupo control $(13,70 \pm 2,08)$ en ninguna de las experiencias.

Los animales que recibieron dietas con un $\mathrm{F} \%=40$ no presentaron un incremento de peso mayor al control, lo que podría deberse a que en modelos animales de obesidad genética e inducida por la dieta, la activación de una respuesta inflamatoria en el hipotálamo perturbando las señales generadas por la leptina e insulina, conduce a un control defectuoso de la ingesta de alimentos y el gasto energético (21-22).

Los grupos $M, O$ y $A O$ tienen un perfil sérico de $A G$ similares, independiente del F\% de las dietas. El perfil sugeriría exacerbación de la ruta de la familia $\omega 9$, con disminución de ácidos linoleico y linolénico. Esto sería consecuencia de la distorsión en el aporte de AG de las fuentes de la dieta.

La dieta $\mathrm{O}$ y $\mathrm{AO}$ aportan grandes cantidades de oleico. Por otro lado, la dieta $M$ aporta ácido palmítico que por la ruta de los AG saturados, luego de procesos de elongación y

\section{TABLA 2}

Consumo de las dietas y VGP de las ratas expresado como media \pm DE.

\begin{tabular}{lcc} 
& CONSUMO $(\mathrm{g} / \mathrm{d})$ & VGP \\
GRUPO C40 & $13,70 \pm 2,08$ & $3,34 \pm 0,30$ \\
GRUPO M40 & & $3,10 \pm 0,29$ \\
F15\% & $13,24 \pm 1,68$ & $3,54 \pm 0,35$ \\
F40\% & $12,94 \pm 1,92$ & $3,27 \pm 0,49$ \\
GRUPO O40 & & $3,20 \pm 0,33$ \\
F15\% & $12,71 \pm 1,89$ & $3,32 \pm 0,27$ \\
F40\% & $10,14 \pm 1,53$ & $3,25 \pm 0,41$ \\
GRUPO AO40 & & $3,38 \pm 0,34$ \\
F15\% & $12,91 \pm 1,54$ & $3,00 \pm 0,32$ \\
F40\% & $12,14 \pm 2,84$ & \\
GRUPO G40 & & $13,75 \pm 2,20$ \\
F15\% & $11,36 \pm 1,43$ & \\
F40\% & & \\
\hline
\end{tabular}

${ }^{*}$ ANOVA -DUNNETT $(\mathrm{p}<0.01)$. 
desaturación, origina ácido oleico.

Las dietas $\mathrm{O}$ y $\mathrm{AO}$ presentan distorsión en la relación $\omega 6 / \omega 3$. La dieta $M$ respecto de las dietas $O$ y $A O$ contiene mayor cantidad de ácido linolénico (w3) y menor cantidad de ácido linoleico ( $\omega 6)$. La dieta M presenta una disminución de ácido araquidónico, perteneciente a la familia $\omega 6$, pero no presenta disminución de DHA, perteneciente a la familia $\omega 3$, que tienen las dietas $\mathrm{O}$ y $\mathrm{AO}$.

En estas tres dietas tiene mayor importancia el tipo de fuente que el F\%, ya que los cambios no se evidencian cuando éste aumenta, pero sí cuando se cambia la fuente de AG.

A diferencia de éstos tres grupos, el G se comporta diferente. Mostró disminuida la ruta de la familia. Cuando el $\mathrm{F} \%$ es elevado $(\mathrm{F} \%=40)$ se observó un aumento de ácido linoleico. En este caso la fuente no fue suficiente para producir cambios, es necesario que esté aportada en altas cantidades. La disminución de DHA, estaría relacionada con la distorsión que presenta la dieta en la relación $\omega 6 / \omega 3$ (FAO-OMS

TABLA 3

Composición de ácidos grasos en suero (\% área media $\pm \mathrm{DE}$ ).

GRUPOS

$M$

$\mathrm{O}$

$\mathrm{AO}$

G

C

M

$\mathrm{O}$

AO

G

c

$M$

$\mathrm{O}$

AO

G

C

$\begin{array}{cc}M & 0,33 \pm 0,16^{*} \\ \text { O } & 0,35 \pm 0,15^{*} \\ \text { AO } & 0,30 \pm 0,16^{*} \\ \text { G } & 0,38 \pm 0,12^{*} \\ \text { C } & \end{array}$

$M$

$\mathrm{O}$

AO

G

C

$\begin{array}{cc}M & 0,71 \pm 0,43 \\ \text { O } & 0,68 \pm 0,24 \\ \text { AO } & 0,12 \pm 0,02 \\ \text { G } & 0,68 \pm 0,21\end{array}$

M

$\mathrm{O}$

AO

G
F\% 15

$20,66 \pm 1,86^{*}$

$19,44 \pm 2,05$

$16,81 \pm 4,24$

$18,07 \pm 1,57$

$19,26 \pm 1,97^{*}$

$21,33 \pm 2,35^{*}$

$24,10 \pm 6,37^{*}$

$11,23 \pm 3,78$

$5,71 \pm 3,35^{*}$

$8,40 \pm 0,96^{*}$

$5,26 \pm 1,48$ *

$18,94 \pm 2,76$

$8,09 \pm 3,33 *$

$17,88 \pm 3,22$

$11,63 \pm 2,28$

$18,47 \pm 3,70$

$0,68 \pm 0,21$

$1,69 \pm 0,63$

$1,08 \pm 0,27 *$

$0,33 \pm 0,20^{*}$

$0,34 \pm 0,05^{*}$
F\% 40

ÁCIDO PALMITICO

$16,08 \pm 2,15$

ÁCIDO OLEICO

$11,29 \pm 2,27$

ÁCIDO LINOLEICO

$9,79 \pm 1,12^{*}$

$14,59 \pm 1,09$

$7,41 \pm 1,89 *$

$17,45 \pm 4,11$

$25,61 \pm 2,42^{*}$

ÁCIDO LINOLÉNICO

$0,44 \pm 0,14^{*}$

$0,28 \pm 0,04^{*}$

$0,32 \pm 0,13^{*}$

$0,28 \pm 0,05^{*}$

$0,81 \pm 0,22$

ÁCIDO ARAQUIDÓNICO

$10,77 \pm 1,14^{*}$

$15,14 \pm 3,13$

$14,47 \pm 2,56$

$20,66 \pm 3,76$

$15,26 \pm 4,95$

ÁCIDO EICOSAPENTAENOICO (EPA)

$1,07 \pm 0,23$

$0,7 \pm 0,27$

$0,75 \pm 0,14$

$0,68 \pm 0,23$

$0,92 \pm 0,19$

ÁCIDO DOCOSAHEXAENOICO (DHA)

$2,76 \pm 0,39$

$0,96 \pm 0,28 *$

$0,39 \pm 0,07^{*}$

$0,40 \pm 0,15^{*}$

$2,22 \pm 0,75$

*ANOVA-DUNNETT $(\mathrm{p}<0.01)$. F\%: Kcal lipídicas/100Kcal totales de dieta. 
recomienda que por cada unidad de AG $\omega 3$ aportados por la dieta, deberían consumirse de 5 a 10 unidades de $\omega 6$ ), el bajo aporte de ácido linolénico y que altos niveles de ácido linoleico pueden inhibir la conversión de ácido alfa linolénico en eicosapentaenoico y docosahexaenoico

Los resultados obtenidos remarcan la influencia de la alimentación sobre el perfil de ácidos grasos séricos y su posible incidencia sobre ciertos factores de riesgo de enfermedades crónicas. En la mayor cantidad de los países industrializados de occidente existe un alto consumo de grasas saturadas y de aceite de girasol. Las grasas saturadas llevan a un aumento de colesterol y triglicéridos (observado en trabajos previos) (23). Ambos caminos llevan a una disminución de los AG de la familia $\omega 3$. Por eso es necesario incorporar otros alimentos que introduzcan en la dieta AG de la familia w3 como pescado, entre otros. El pescado, en especial el pescado azul, es una fuente muy importante de ácidos grasos omega 3, con demostrados efectos beneficiosos en la salud cardiovascular y en el desarrollo cerebral.

Por ello, no sólo es importante tener en cuenta el porcentaje de lípidos de las dietas consumidas, sino también los diferentes ácidos grasos que la componen, teniendo en cuenta las recomendaciones internacionales.

\section{RESUMEN}

El perfil de lípidos de una dieta es importante para prevenir enfermedades crónicas y mejorar la calidad de vida de los individuos. Objetivo: estudiar el efecto de dietas con diferentes fuentes y concentraciones lipídicas sobre el perfil de ácidos grasos en suero de ratas en período de crecimiento activo. Las dietas experimentales, contenían 15 y $40 \mathrm{Kcal} \%$ de lípidos aportados por: manteca $(M)$; aceite de oliva $(O)$; aceite de girasol alto oleico (AO) y aceite de girasol (G). El control recibió dieta normocalórica (fuente lipídica: aceite de soja). Los grupos $\mathrm{M}$, O y AO presentaron un aumento en los niveles séricos del ácido oleico, con disminución de los ácidos grasos esenciales (AGE) con respecto al grupo C. Estos resultados se observan independientemente del F\% de la dieta. El grupo $\mathrm{G}$ muestra aumento de ácido linoleico sólo cuando el F\% de la dieta fue 40. La administración de estas dietas provocó cambios en el perfil de ácidos grasos en suero en respuesta a las diferentes fuentes aportadas por las dietas, sólo algunos cambios observados en el grupo $\mathrm{G}$ fueron también en respuesta al alto porcentaje de grasa.

Palabras clave: Nutrición; ácidos grasos; lípidos; aceites; grasa láctea; ratas.

Agradecimientos: Financiado por UBA N²0020120200068

\section{BIBLIOGRAFÍA}

1. Estrategia Mundial sobre Régimen Alimentario, Actividad física y Salud-WHO, 2004.

2. Burrows, R. Prevención y tratamiento de la obesidad desde la niñez: la estrategia para disminuir las enfermedades crónicas no transmisibles del adulto, Rev Méd Chil. 2000; 128:105-10.

3. Harika RK. Cosgrove MC. Osendarp SJ. Verhoef P. Zock PL. Fatty acid intakes of children and adolescents are not in line with the dietary intake recommendations for future cardiovascular health: a systematic review of dietary intake data from thirty countries, Br J Nutr. 2011; 18:1-10.

4. Perris P. Fernandez I. Feliu MS. Gurevich M. Boschi E. Kordich L. Bordoni N. Slobodianik N. Perfil lipídico en un grupo de niños en riesgo nutricional. Actualización Nutr. 2006; 7: 72-4.

5. Burr G.O. Burr M. M. A New Deficiency Disease Produced by the Rigid Exclusion of Fat from the Diet. J Biol Chem. 1929; 82:345-67.

6. Erkkila A. de Mello VD. Riserus U. Dietary fatty acids and cardiovascular disease: An epidemiological approach. Prog Lipid Res. 2008; 47:172-87.

7. Jicha Gregory A. Markesbery William R. Omega-3 fatty acids: potential role in the management of early Alzheimer's disease, Clin Interv Aging 2010; 5: 45-61.

8. Chicco Adam J. Sparagna Genevieve C. Linoleate-rich highfat diet decreases mortality in hypertensive heart failure rats compared to lard-rich and low-fat diets. Hypertension 2008; 52(3): 549-55.

9. Anandan C. Nurmatov U. Omega 3 and 6 oils for primary prevention of allergic disease: systematic review and metaanalysis. Allergy 2009; 64(6):840-8.

10. De Spirt S. Stahl W. Tronnier H. Intervention with flaxseed and borage oil supplements modulates skin condition in women, Br J Nutr. 2009; 101(3):440-5.

11. Surette ME. Stull D. The impact of a medical food containing gamma-linolenic and eicosapentaenoic acids on asthma management and the quality of life of adult asthma patients. Curr Med Res Opin. 2008; 24(2):559-67.

12. Simopoulos AP. Omega-3 fatty acids in inflammation and autoimmune diseases, J Am Coll Nutr. 2002; 21:495-505.

13. Schirmer MA. Phinney SD. Gamma-linolenate reduces weight regain in formerly obese humans, J Nutr. 2007; 137(6):1430-5.

14. Stanley JC. Elsom RL. Calder PC et al. UK Food Standards Agency Workshop Report: the effects of the dietary n- 6:n3 fatty acid ratio on cardiovascular health. British J Nutr. 2007; 98:1305-10.

15. Simopoulos A.P. The importance of the ratio of omega- 6/ omega-3 essential fatty acids, Biomed Pharmacoth. 2002; 56 (8):365-79.

16. Pérez-Jiménez F. Ruano J. Perez martinez P. Lopez-Segura F. Lopez-Miranda J. The influence of olive oil on human health:not a question of fat alone, Mol Nutr Food Res. 2007; 51(10): 1199-208.

17. Waterman E. Lockwood B. Active components and clinical applications of olive oil, Altern Med Rev. 2007 12(4):33142.

18. Reeves $P G$, Nielsen FH. AIN-93 purified diets for laboratory rodents: final report of the American Institute of Nutrition ad hoc writing committee on the reformulation of the AIN-76A rodent diet, J Nutr. 1993; 123(11):1939-51.

19. Guy Lepage. Claude C. Roy. Direct transesterification of all classes of lipids in one-step reaction. J Lipid Res. 1986; 27:114-20.

20. Winer B. J. Brown. Statistical principles in experimental design. McGraw-Hill Book company. 1991.

21. Milanski M. Degasperi G. Saturated fatty acids produce an inflammatory response predominantly through the activation of TLR4 signaling in hypothalamus: implications for the pathogenesis of obesity. J Neurosci. 2009; 29(2): 359-70.

22. Velloso LA. Araújo EP. de Souza CT. Diet-induced inflammation of the hypothalamus in obesity. Neuroimmunomodulation 2008; 15 (3):189-93.

23. Perris P. Fernandez I. Impacto de dietas con alto contenido en grasa sobre el perfil de lípidos séricos: estudio en ratas en crecimiento. Actualización Nutr. 2012; 13 (3): 185-90. 